\title{
LA PRIMERA FERTILIZACIÓN IN VITRO EXITOSA EN EL INSTITUTO NACIONAL MATERNO PERINATAL. MAYO 2015
}

\author{
Marco Garnique Moncada1', Ruth Barrientos², Antonio Cipriano Bernuy³, Luis Carpio Guzmán4, \\ Jorge Rojas Tenorio ${ }^{5}$, José Quispe ${ }^{5}$, Ángelo Torres ${ }^{5}$, Isela Mancisidor ${ }^{2}$, Úrsula Guevara ${ }^{6}$
}

\begin{abstract}
RESUMEN
La Fertilización in Vitro (FIV), es un procedimiento de Reproducción Asistida de alta complejidad que utilizamos en el manejo de la pareja infértil. El objetivo del presente artículo es la difusión de la primera fertilización in vitro en una Institución Pública como lo es el Instituto Nacional Materno Perinatal, con resultado de nacimiento de 2 Recién Nacidos (RN) normales.
\end{abstract}

Palabras clave: FIV; Transferencia embrionaria; Institución pública (Fuente: DeCS BIREME).

\section{FIRST SUCCESSFUL IN VITRO FERTILIZATION AT THE NATIONAL MATERNAL PERINATAL INSTITUTE. MAY 2015}

\begin{abstract}
In Vitro Fertilization (IVF) is an assisted reproduction procedure of high complexity that we use in the management of the infertile couple. The aim of this article is the dissemination of the first IVF in a public institution such as the National Maternal and Perinatal Institute, resulting in the birth of two normal newborns.
\end{abstract}

Key words: IVF; Embryo transfer; Public institution (Source: MeSH NLM).

\section{INTRODUCCIÓN}

No solo en nuestro país sino que en muchos lugares del mundo, la postergación de la maternidad es algo natural y comprensible en nuestros tiempos y está relacionada principalmente al cambio de la dinámica social, profesional y laboral de la mujer contemporánea; la mujer de hoy busca alcanzar sus metas profesionales y laborales dejando el embarazo postergado, temiendo que esta pueda interferir en su desarrollo profesional. La maternidad se sacrifica reduciéndola o posponiéndola por tener una mejor calidad de vida en el futuro. Lamentablemente el tiempo, es decir la edad es el enemigo número uno de la fertilidad de la mujer no sólo porque después de los 35 años existen un menor número de óvulos y la calidad de los mismos disminuye sino que además se presentan una serie de enfermedades ginecológicas, metabólicas que ensombrecen más aún el pronóstico reproductivo.

Dentro de la atención a la salud reproductiva en la mujer y su pareja, se considera una serie de acciones que contribuyen al logro del bienestar físico, mental y social y no a la mera ausencia de enfermedades o dolencias relacionadas con el sistema reproductivo y sus funciones o procesos. En consecuencia la salud reproductiva entraña la capacidad de disfrutar de una vida satisfactoria y sin riesgos de procrear, así como la libertad para decidir hacerlo o no, cuando y con qué frecuencia.

La iniciativa del Proyecto de Mejora en el "Manejo integral de la pareja infértil en la Unidad de Infertilidad del Instituto Nacional Materno Perinatal" que se presentó en el año 2012, se vio cristalizada cuando nacieron 2 Recién Nacidos sin patologías el 5 de Mayo del 2015, hecho que marcará un hito en la ginecología de las instituciones del Estado por ser la primera vez que una paciente es tratada en su totalidad y con resultados exitosos mediante Tecnología de Reproducción Asistida de Alta Complejidad como la Fertilización In Vitro en una Institución Pública como lo es el Instituto Nacional Materno Perinatal (INMP).

\section{DESCRIPCIÓN DEL PROCEDIMIENTO}

Fue nuestra tercera paciente la Sra. ACM, quien resultaría en la culminación exitosa de dos recién nacidos sanos. Nuestra primera paciente no embarazo y la segunda paciente tuvo un embarazo bioquímico, es decir HCG (hormona gonadotropina coriónica) positivos con valores bajos y en descenso.

\footnotetext{
Médico Gineco-Obstetra, Asistente del Servicio de Medicina Reproductiva del Instituto Nacional Materno Perinatal. Master en Reproducción Humana. Profesor de la Facultad de Medicina de Universidad Nacional Mayor de San Marcos. Lima-Perú.

Bióloga, Servicio de Medicina Reproductiva.Instituto Nacional Materno Perinatal. Lima-Perú.

Médico Gineco-Obstetra, Jefe de Servicio de Medicina Reproductiva del Instituto Nacional Materno perinatal. Lima-Perú.

4 Médico Gineco-Obstetra, Jefe de Servicio de Ginecología Especializada del Instituto Nacional Materno perinatal. Lima-Perú.

Médico Gineco-Obstetra, Asistente del Servicio de Medicina Reproductiva del Instituto Nacional Materno perinatal. Lima-Perú.

Lic. Enfermería del Servicio de Medicina Reproductiva. Instituto Nacional Materno Perinatal. Lima-Perú.
} 


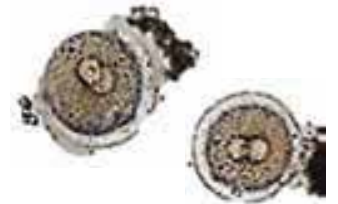

La paciente ACM de 33 años quien padecía de Infertilidad por Factor Tubárico Irreparable quirúrgicamente tenía un tiempo en busca de embarazo de 5 años. Como datos importantes en la historia clínica se reporta ser GOPO, Menarquia a los 13 años, régimen catamenial 3 de 30 días y como antecedente patológico tenía TBC genital con tratamiento completo y reserva ovárica disminuida. Antecedentes quirúrgicos una Laparoscopia diagnóstica. Su pareja VEGH de 29 años, sano y sin hijos.

La paciente fue sometida a una estimulación hormonal para alta complejidad en esquema con Antagonistas, Maduración final ovocitaria con HCG y aspiración ovocitaria a las 36 horas en Sala de Operaciones (SOP) N8, obteniéndose 4 ovocitos en estadio MII (adecuado grado de maduración) el 3 de setiembre del 2014 a las 10:40 hr. Los ovocitos fueron fecundados mediante Fertilización Vitro en el Laboratorio de Gametos y Embriones en nuestro instituto, de los cuales sólo dos embriones llegaron al día 3 con un desarrollo embrionario adecuado, los cuales mediante un catéter de Transferencia

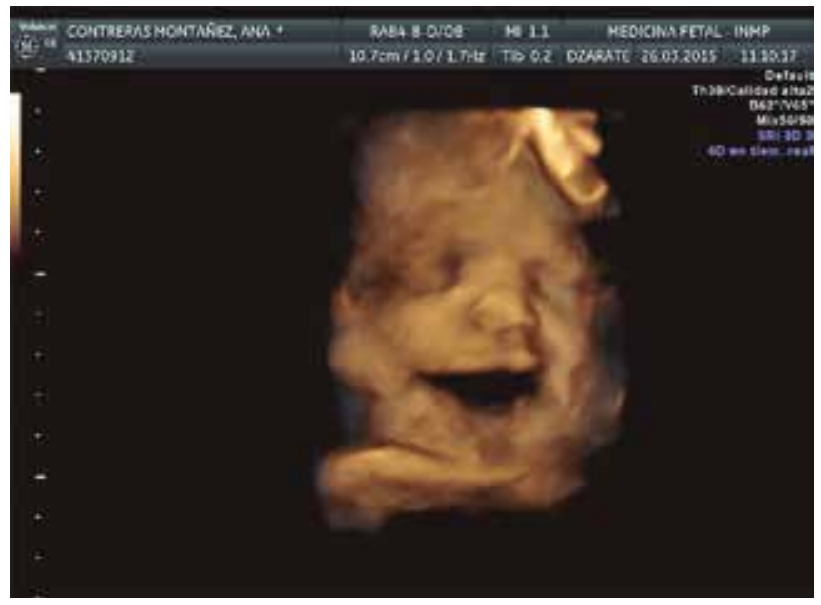

Embrionaria fueron colocados con técnica depurada y bajo guía ecográfica en el tercio medio de la cavidad uterina el 9 de setiembre del 2014 a las 9:20 hr, en SOP N 8 del INMP.

Dos semanas después la paciente es evaluada mediante resultado de HCG Beta cuantitativo resultando 178 $\mathrm{UI} / \mathrm{ml}$, lo que confirmaría su embarazo y con ello una evaluación posterior mediante ecografía transvaginal el 6 de Octubre del 2014, confirmándose una gestación doble activa bicoriónica, la evaluación embrionaria iría bien al confirmase un desarrollo adecuado para el 20 de octubre. Para el 14 de noviembre se le realizó la ecografía genética en el Servicio de Medicina Reproductiva y todos los parámetros de la evaluación fueron normales, Así mismo

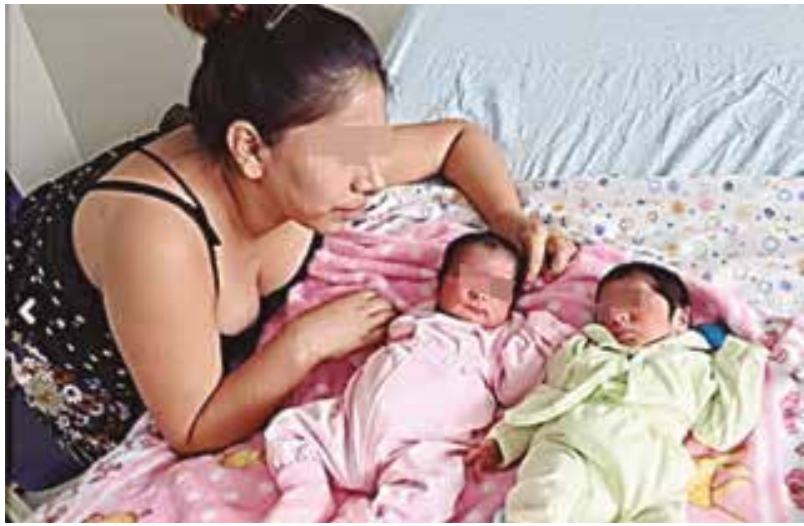

se realizó una ecografía morfológica a las 22 semanas reportándose que el desarrollo de los fetos estaban acorde con las semanas de gestación y sería un varón y una mujer, para satisfacción de los padres. Durante todo el control prenatal se tuvieron los cuidados respectivos, tratando de vigilar y prevenir siempre la prematuridad a la que están expuestos los embarazos múltiples, ya con 35 semanas y con un peso promedio de 2500 gr. Se le aplico sus dos dosis de betametasona intramuscular, programándose la cesárea a las 36 semanas por dinámica esporádica y feto 2 en Transverso Izquierdo.

La cesárea fue realizada con éxito y sin complicaciones el 5 de Mayo del 2015 por el Dr. Marco Garnique Y Dr. Antonio Cipriano, los mismos y que junto con los Biólogos Ruth Barrientos, Ángelo Torres e Isela Mancisidor y todo el equipo de Medicina Reproductiva que trabajó desde el inicio con las pacientes incluidas en el programa de Fertilización In vitro. Esto permitió EI Nacimiento del RN1 masculino con 2400 gr y apgar $8^{1}$ y $9^{5^{\prime}}$ y de la RN2 femenino con $2746 \mathrm{gr}$ y con apgar $8^{1}$ y $9^{5}$ a horas $10: 42$ y 10:43 respectivamente, ambos con buen estado de salud y dados de alta al tercer día de la cesárea junto con la madre.

El premio Nobel de Fisiología y Medicina 2010 y pionero en éste tipo de tratamientos el Fisiólogo Robert Edwars y el ginecólogo Patrick Steptoe realizaron este procedimiento con éxito en año 1978, por lo que somos conscientes del camino ya recorrido por la Medicina Reproductiva, sin embargo estamos fortalecidos con éste logro en el INMP ya que es una institución pública y con muchas ganas de seguir contribuyendo con el apasionante mundo de la Medicina Reproductiva, hoy por hoy una de las especialidades con mayor desarrollo y retos en la Medicina Moderna.

Finalmente resaltar que el Servicio de Medicina Reproductiva del INMP brinda esta tecnología al alcance de las parejas de menores recursos económicos del país; de esta manera la sociedad verá acortada la brecha de la desigualdad cuando se le exponga que tiene la oportunidad ser tratada y sus expectativas colmadas ya que una institución pública asume el gran reto de tratarla.

Conflictos de interés: Ninguno. 


\section{REFERENCIAS BIBLIOGRÁFICAS}

1. MATARAZZO J (1980). Behavioral health and behavioral medicine: frontiers for a new psycology. Amer Psych; 35(9):807-817.

2. Hervey S. Chandra A. Declining estimates of infertility in the United States: 1982-2002. Fertil \& Steril 2006; 86: 516-23.

3. Society for Assisted Reproductive Technology. American Society for Reproductive Medicine. Assisted reproductive technology in the US: 2000 results generated from the American Society for Reproductive Medicine/Society for Assisted Reproductive Technology Registry. Fertil Steril. 2004; 81(5):1207-20.

4. Remohí y col. Manual Práctico de Esterilidad y Reproducción Humana. 2012.
5. Ysis Roa Meggo. Los Psicólogos y la Infertilidad. Psicología y salud, enero-junio, 2008, vol 18: 129-135

6. Ysis Roa Meggo. La Infertilidad en el Perú. Lima 2009.

7. Busso N, Pellicer A. (2014) Inducción de la Ovulación (2 ${ }^{\text {da }}$ ed.). Barcelona-España: Elseiver.

8. La República. (8 de Mayo 2015). Nacen los primeros mellizos por fertilización in vitro en Maternidad. Recuperado de http:// www.larepublica.pe/

Correspondencia: Marco Antonio Garnique Moncada Dirección: Avenida Miroquesada N651-Lima 17 Teléfono: 997361014

Correo electrónico: marcogarnique@yahoo.es 